\title{
A Survey of Routing Protocols for Low Power Sensor Networks
}

\author{
Kulsoom Fatima \\ Department of Computer Science \\ The University of Lahore, Sargodha Campus
}

\author{
M. Mateen Yaqoob \\ Department of Computer Science \\ The University of Lahore, Sargodha Campus
}

\begin{abstract}
A Wireless Body Area Sensor Network (WBASN) is combination of a set of sensor nodes, placed on/near or inside a human body. Wireless Body Area Sensor Networks (WBASN) is a developing technology which exploits wireless sensor nodes to implement real-time wearable health monitoring of patients to enhance independent living. These sensor nodes can be worn externally to monitor multiple bioparameters (such as blood oxygen saturation $\left(\mathrm{SpO}_{2}\right)$, blood pressure and heart activity) of multiple patients at a vital location in the hospital. Hence the mission of the WBASN is very critical, increasing the lifetime of nodes is essential in order to maintain both practicality and effectiveness. There are number of routing protocols for WBASN. This paper will discuss the architecture of WBASN. Moreover, this work gives detailed literature review of many different routing protocols belongs to the field of WBAN and also discuss their strengths and weaknesses.
\end{abstract}

\section{Keywords}

Network lifetime, Health monitoring, Multi-hop, Energy consumption, Routing protocols, WBASNs.

\section{INTRODUCTION}

A wireless sensor network (WSN) is discussed as a distributed network having dispersed and autonomous sensing station. Each single sensing station is said to a sensor node. A sensor node comprise of a microcomputer, transceiver, and a power source(mostly a battery) and depending upon the application area also use some sensor(s). Few smart sensor prepared with an electro-mechanical device, which is used to control the different components of the established system, known as actuator[1].After formation of a network, sensor nodes sense, measure, and collect information of any activity from surroundings and report the sensed data to the base station in multi-hop fashion.

Now a day Wireless Sensor Networks (WSNs) are being used to monitor different parameters in many applications from environment monitoring, to habitant monitoring, [2] war field, farming and smart homes. These wireless sensors are scattered in sensing area to keep care full check on field. WBAN is new emerging sub-category of WSN. A key application of WBAN is health monitoring.

Patient monitoring is developing as an important application of embedded sensors network. Many wireless sensors are implanted in-body or on-body of the patient. These tiny wireless sensors make Wireless Body Area Sensor Networks (WBASNs). WBASNs can continuously monitor physiological conditions of patient under supervision, and in case of emergency can provide real-time feedback. Through WBASN a patient is constantly monitored, and in case of some critical situation an immediate action should be required. These sensors can collect the physiological data and then send to physician in a hospital via Metropolitan Area Network (MAN) or Local Area Network (LAN). Where, diagnosis from received information is performed and on that base decisions are taken. WBASNs are used for medical and non-medical applications [3].Wireless sensors are placed on the human body or embedded in the body to monitor vital signs like blood pressure, body temperature, heart rate, glucose level etc. Use of WBAN technology to monitor health parameters significantly declines expenditures of patient in hospital.

According to the reports of Department of Economics and Social Affairs of United Nation Secretariat [4] people of age 65 and over, which account for almost 15 percent of world population, will nearly double and became 761 million by the 2025. This fact reveals that by the mid-century the medical care will become a main issue. As the old aged people are more susceptible to many health related diseases and issues, so they have need of instant medical care and checkup, which results in high health care budget[5,6].Theses facts and figures claim major modification and proactive management which focus on the avoidance, prevention and early treatment of multiple diseases[7] .

With the assistance of WBAN technology, patients are monitored at home for extensive period. Sensors uninterruptedly sense data and advancing to medical superintendent. WBANs having the issue that sensor node are functioned with inadequate energy source. It is requisite to use least power for transmitting data from sensor nodes to sink. One of the foremost complications in WBAN is to recharge the batteries. An efficient routing protocol is required to overcome this issue of recharging batteries. Numerous energy effectual routing protocols are suggested in WSN technology [8], [9], and [10]

However, WSNs and WBANs have different manners, applications and operate in different conditions. The sensor nodes of WBASNs have different energy level and also produce different sort of data. While the sensor nodes of wireless sensor network (WSNs) have almost equal level of data rate and similar energy level. It is difficult to implement WSN routing protocols to WBAN. Consequently, energy proficient routing protocol for WBAN is requisite to monitor patients for extensive period.

In this paper, section II describes the brief architecture of WBASNs. Section III provides background information related to this topic. In section IV reviews of different researches is presented. At the end section $\mathrm{V}$ concluded the whole work. 


\section{ARCHITECTURE OF WBASNS}

In body area network (BAN) a node is denoted as an independent device having communication capability depending upon their application and functionality, and part in network, BAN nodes are categorized into different groups. In term of functionality there are three types of node available.

(i) Sensor; which measures definite parameters inside or within one's body and then after gathering and processing necessary data provide wireless response to information. (ii) Actuators; once receives data from sensor it established link with the user [11].

(iii) Personal Device; handles interaction with other users and also gathers whole information which is received from sensor and actuators

In the sense of implementation sensors nodes can be categorized into three different classes, which are Implant Node, Body surface node and External node. One of them are embedded in the human body, $2 \mathrm{~cm}$ away from it or far away from body respectively $[12,13]$. Based on their working in the WBAN sensor nodes can be classified into following categories;

a- Coordinator: It acts as a gateway $(\mathrm{GW})$ to the external world or any other BAN. b- End Node: End node is only capable of accomplishment of their implanted application. c- Routers: A Parent node and child nodes have nodes which act as an intermediate node through which they relay messages called routers.

Based upon IEEE 802.15.6 standard BAN nodes are supposed to function in either single hope or multi hop star topology in which central node can be placed on a body position like waist $[13,14]$.With respect to communication architecture WBAN can be alienated into three different tiers. For enabling an efficient and module based system this communication architecture covers many design issues. As shown in figure 1, in a centralized network architecture devices of WBAN are distributed throughout the human body where the exact location of any device is depend upon the application.

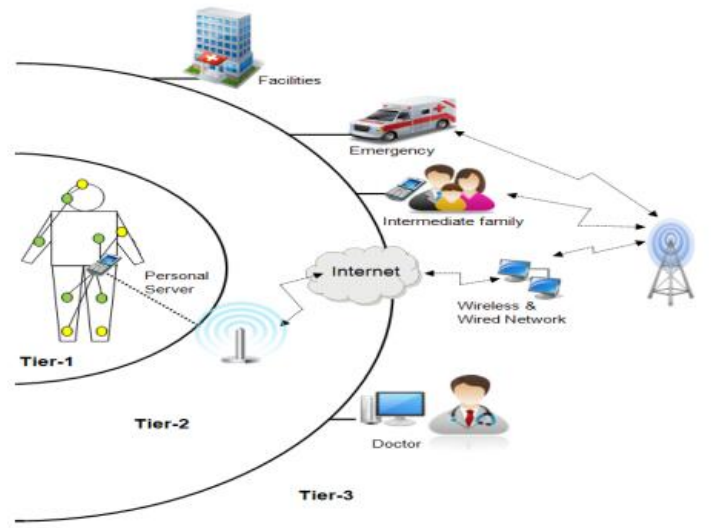

Figure 1: Architecture of Wireless Body Sensor Network [15]

The architecture of WBASNs can be divided as following three different tires

- Tier 1: In tier 1 the on body and implanted bio medical sensor nodes send the sensed data to the coordinator or base station.
- Tier 2: In Inter-WBSNs, after required data processing and aggregation, coordinator or base station send the received data to the $\operatorname{sink}(\mathrm{s})$.

- Tier 3: At this level the $\operatorname{sink}(\mathrm{s})$ send the collected data to the isolated medical center or any other destination via established network. [16]

\section{MOTIVATION AND BACKGROUND}

Wireless Body Area Sensors are used to monitor human health with inadequate energy resources. Different energy efficient routing patterns are used to forward data from body sensors to medical server in hospitals. It is important that sensed data of patient consistently received to medical specialist for more analysis.

The increasing temperature of implanted sensor nodes due to communication radiations and circuitry power feeding can affect the human body. In [17], authors use thermal-aware routing to decrease the effect of rising temperature of implanted sensor nodes. Quwaider et al. [18] used Single-hop communication and enlarged transmission range of sensors to overwhelmed problem of partitioning. A protocol named Environment Adaptive Routing (EAR) algorithm [19] defines different communication cost for heterogeneous WBASNs devices. Though, the Single-hop communication and preemptive routing are not suitable choices for WBASNs. Multi-hop communication is appropriate for normal packet delivery and due to high transmission cost only Single-hop is used for emergency services. However, after a steady interval use of Hello messages at this point, results in high energy feeding.

In [20], Wireless Autonomous Spanning Tree Protocol (WASP) is defined to achieve low delay and increased network reliability for WBASNs. In WASP-scheme a message is distributed to update parent nodes with information of its child nodes. However, power balancing issue is not discussed. Annur et al. [21] applied tree algorithm with prioritization for WBASNs which can be applied in medical and non- medical fields. In this paper a prioritization for emergency case for both medical and non-medical WBANs application is presented. Where, a channel is dedicated for emergency data delivery and normal data transmission is lagged until the successful delivery of critical data. However, the dedicated channel results in loss of available resources. A collision resolution protocol, Tree algorithm is used to achieve the goal. This algorithm allows the high priority nodes to get the channel first without competing low priority nodes.

\section{ROUTING PROTOCOLS}

In wireless body area sensor network (WBASN) technology, large numbers of routing schemes are proposed. In this section, some proposed routing protocols are presented.

\subsection{A New Energy-Efficient routing Protocol (M-ATTEMPT)}

M-ATTEMPT [3] is an energy efficient and thermal aware routing protocol for WBSNs. It decreases the nodes temperature in addition to this also minimize the delay for the acute data using heterogeneous bio-medical sensor nodes. In the network design of this arrangement, high data rate nodes are positioned at less movable places of the human body whereas the sink node act as base station (BS) is positioned at the middle. For critical or request driven data packets the sensor nodes of this scheme enhance their communication power to advancing the data packets directly to the sink node 
(single-hop). On the other hand, for ordinary data packets delivery multi-hop communication is used for this purpose. The sensors which have ordinary data packets cannot forward them until the sink (base station) received all the critical or request driven data packet. While in case of multi-hop communication, a route with minimum hop-count is carefully chosen if two or multiple routes are accessible.

If multiple next-hop neighbor nodes have the similar hopcount then the neighbor node with minimum energy consumption to the sink is chosen. To control temperature increase issue, M-ATTEMPT states a threshold value and if any node's temperature goes elsewhere that value, it breakdowns all the possible routes with the neighbor node. Though, if the node's temperature grasps to the threshold value after getting a data packet, it again send that packet to preceding node and the preceding node show it as a hotspot.

The four diverse phases of M-ATTEMPT include: (i) initialization phase, (ii) routing phase, (iii) scheduling phase, and (iv) data transmission phase are shown in figure 2 . At start in initialization phase all sensor nodes send out the hello packet, whereas in second phase the routing phase routes with minimum hop-counts are chosen within the existing routes based on above-mentioned procedure. The sink node (BS) produces a Time Division Multiple Access (TDMA) schedule for all root nodes in the scheduling phase, while throughout the data transmission phase the root nodes send their data to the sink node.

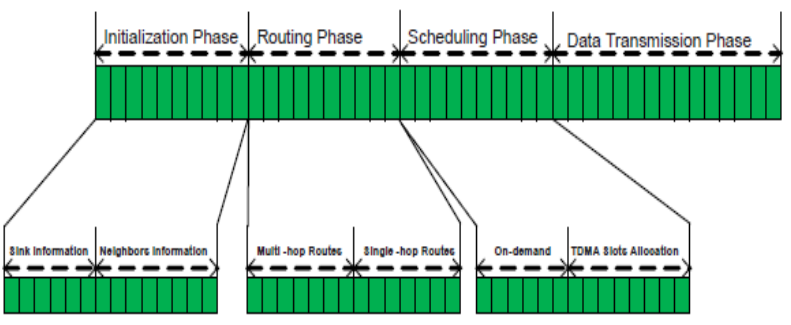

Figure 2: Sequences of phases in each round [3]

\subsection{Wireless Autonomous Spanning Tree Protocol (WASP)}

In [20] the authors proposed a cross-layered protocol, named as wireless autonomous spanning tree protocol. WASP distributes the time axis into slots in dispersed manner, known as WASP-Cycles. A WASP-Cycle deliver traffic routing and medium access coordination using the same spanning tree which results in lower energy consumption and higher throughput The spanning tree is set up automatically as shown in Figure 3. This spanning tree is used to send the data to the sink, and integrates the medium access control and routing.

A unique WASP scheme message is assign to each node which sends it to its child nodes informing them when they are allowed to use the link. Therefore, these messages permit traffic control and rise resource request from parents of children which minimize the coordination overhead. Based on the sink WASP-scheme and the child node's requirements the children respond to the scheme by transmitting their own
WASP-scheme. It is essential to know that nodes are synchronized to avoid shifting.

For sink and child nodes, WASP-scheme messages are different but they typically comprise of the following: i) address of sender node (ii) slots already allocated to the children of the parent node where they send their WASPscheme (iii) silent period interval (iv)forwarding received data to the sink (v) Contention slot (vi) Acknowledgment order .

Results show that WASP accomplishes almost 94\% throughput, maximum packet delivery ratio, little energy consumption and stable end to end delay. Though, WASP does not consider quality of link, mobility, and load and also does not support interactive communication. Overhead is also a drawback which can be minimizing with data aggregation procedures.

\subsection{Cascading Information Retrieval by Controlling Access with Dynamic Slot Assignment (CICADA)}

Latre et al. [22] proposed A Secure Low-Delay Protocol for Multi-hop Wireless Body Area Networks (CICADA) routing protocol which consists of a spanning tree structure same as WASP [20] as shown in figure 3. CICADA is a cross-layered low energy protocol used Time Division Multiple Access (TDMA) scheduling which is based on multi-hop mobile body area networks. In it Time Division Multiple Access (TDMA) protocol schedules the transmission of nodes. Slots of the time are assigned in scattered custom and the identified length of each cycle makes the slot synchronization promising.

In this protocol parents nodes are responsible for telling their children when they can communicate. The nodes near the root act as forwarder nodes or parent nodes, these nodes collect data from their associated children nodes and relay to sink. Due to additional traffic load of children nodes on parent nodes causes parent nodes to exhaust their energy fast.

This scheme divides each cycle in to sub-cycle: one is the control sub cycle and second is data sub-cycle. Each subcycle assigns the slots allowing to its own scheme: the control scheme and the data scheme, and during the control sub-cycle from the parent nodes to their children nodes both are forwarded. Each control scheme demonstrates the order, in which the children node can send their parents their control scheme, the length of control sub cycle, and also depth of the tree.

The data sub-cycle starts after the termination of the control sub-cycle. The length of waiting period and the length of data period are the contents of data scheme. In the aforementioned data period, the child node sends data packet to their parent node. To save power, throughout the waiting period node can also go to sleep mode. Each parent node builds a table of its children nodes holding the sum of slots they need to transfer the data to their parent node and the number of slots mandatory to receive the data from their children nodes. To join the tree each data sub-cycle has a slot for new nodes. After hearing the data scheme of the desired parent node, each new child node is allowed to send JOIN-REQUEST message in that slot. 

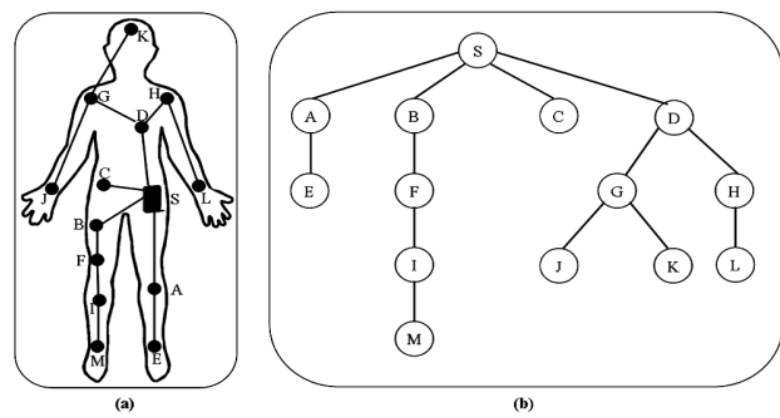

Figure 3. (a) On-body Network (b) Abstract view of the network [16]

\subsection{Distance Aware Relaying Energy Efficient (DARE) Protocol}

In [23] the authors said that WBASN playing a significant role to get the real time and precise data with reduced level of energy consumption. WBASN comprises of tiny, lightweight and energy restricted sensors, placed in or on the human body, to observe any uncertainty in body tissues and measure numerous biomedical parameters related to patient. In this research a protocol named Distance Aware Relaying Energy efficient (DARE) to monitor patients in multi-hop Body Area Sensor Networks (BASNs) is proposed. The presented protocol functions by scrutinize the ward of a hospital containing of eight patients. They are under different topologies by putting the sink at different locations in ward or fabricating it fixed or movable.

In a hospital scenario seven sensors including Body Relay (BR), continuously data monitoring sensors (BS) and eventdriven data (Threshold) monitoring sensor (BS) are attached to each patient. These sensor nodes measures different parameters related to patient's health which includes Electrocardiogram (ECG), pulse rate, heart rate, temperature level, glucose level, toxins level and motion. To decrease the energy consumption, these sensors creates a link with the sink by means of an on-body relay, attached on the chest of each patient. The attached body relay retains greater energy resources as compared to the body sensors as; they perform amassment and relaying of data to the sink node. Hence, for heterogeneous networks; a relaying energy-efficient protocol for constantly observing patients is suggested. In the given scenarios, few sensors nodes constantly monitor patient's data whereas, some other nodes monitor data only when they notice a definite threshold stage. The protocol defines minimum energy parameters for the sensors to avoid damage to the sensitive body tissues of human body. Results of the research depict greater packet delivery ratio, longer network life time and better stability period but it has high propagation delay.

\subsection{Opportunistic Routing}

In [24], the authors have recommended an opportunistic routing protocol by bearing in mind the stirring nature of the human body. The network model used in this protocol is very simple, where a bio-medical sensor node on the chest, senses the data and sends it to the other node i.e. sink node which is on the wrist of the patient.

Furthermore, there is a relay node on the wrist to simplify the communication between the bio-medical sensor node and the sink node. During movement (walking or running) of human body the wrist (having the sink and relay nodes) moves onward and backward causing into two types of promising communication establishment: (a) None Line of Sight (NLoS) communication - when the wrist is at the back of the body and (b) Line of Sight (LoS) communication when the wrist is at the front of the body. Both None Line of Sight (NLoS) and Line of Sight $(\mathrm{LoS})$ communications are reflected to have the same probability, which is almost 0.5 . The sensor node sends Request to Send (RTS) packet, if it has data packets to send to sink node, this will only be received by LoS nodes. If the RTS packet is recognized within a certain time interval, i.e., the sink node is in LoS with sensor node, then the sensor node starts promoting the data packets directly to the Sink.

On the other hand, the sensor node will send a wakeup packet to relay node if, the RTS packet is not recognized within definite time of interlude, i.e., the sink node is in NLoS with the sensor node. Once the relay node is ready for communication, it will notify both the sensor node and sink node to initialize communication. After successfully receiving all the data packets, the sink node will send a Receive Acknowledge (RACK) packet to the sensor node. If the sensor node does not receive the RACK packet, then the abovementioned practice will be recurring until successful communication.

\subsection{Hybrid Indirect Transmission (HIT)}

Culpepper et al. [25] proposed a data gathering protocol based named Hybrid Indirect Transmission (HIT). This protocol is based on one or more than one cluster, where each cluster is capable of several multi-hop transmissions. To reduce energy absorption and the network delay HIT uses parallel processing both in between-cluster and within-cluster communication. In this research, analysis oh HIT and HITm (HIT having multiple clusters) shows minimum network delay, increased network life time and greater energy efficiency.

The detail procedure of HIT is as follows: In the initial stage, one or many cluster heads $(\mathrm{CH})$ are selected. Then throughout the network cluster heads $(\mathrm{CH})$ send out their status information.in next step upstream and downstream relation of $\mathrm{CH}$ are established. Therefore, multiple routes are structure within a cluster to the $\mathrm{CH}$. Then each node computes its blocking set. The blocking set of node i consists of the list of nodes that are not permitted to transmit instantaneously with node $i$. More precisely, node $i$ blocks node $\mathrm{j}$ only if

$$
D(i ; u i)>D(i ; u j)
$$

In this equation upstream neighbor of node $\mathrm{j}$ is uj. At present, a Time Division Multiple Access (TDMA) schedule is calculated for each node which permits extreme communication between nodes with comparable transmissions. At the end, nodes transmit to their upstream neighbors over the TDMA schedule formerly allocated. Unfortunately, HIT needs extra communication power in dense networks. For specific applications and its communication routes HIT does not deliberate reliability and has conflicting interaction issues amongst the desired routes.

\subsection{Mobile Sink Improved Energy Efficient PEGASIS-based Protocol (MIEEPB)}

In [26] an improved version of Power-Efficient Gathering in Sensor Information Systems (PEGASIS) is presented. This paper gives the idea of mobility of a sink in improved energy efficient PEGASIS-based protocol (IEEPB) to development the network lifetime of Wireless Sensor Networks (WSNs). The idea of multi-head chain, multi-chain and the sink 
Mobility greatly influence in increasing the network lifetime of wireless sensor nodes. The recommended idea of Mobile sink improved energy-efficient PEGASIS-based routing protocol (MIEEPB); is a multi-chain model which provide mobility of sink, to attain effective energy usage of wireless sensors. There is a need to enclose its movement within boundaries and the route of mobile sink should be static as the automated shifting of mobile sink is conduct by petrol or current.

In this technique, the mobile sink moves along its route and stays at sojourn location for a sojourn time to give affirmation of comprehensive data collection. An algorithm has developed for route of mobile sink and also performing wide-range of experiments to evaluate the achievement of the advised approach. The outcomes of this research declares that proposed scheme is almost excellent and also performs exceptional than IEEPB in terms of network lifetime and stability period. It controls the average energy consumption of nodes, and shrinks the delay in data delivery by means of smaller chains.

\section{COMPARATIVE REVIEW OF ROUTING PROTOCOLS}

The aforementioned protocols can be classified into different types of routing protocols. The researchers divided them in to temperature aware routing protocols, cluster based routing protocols, postural movement based routing protocols, and cross layered based routing protocols.

M-ATTEMP [3] is a temperature aware routing protocol. As compared to the concept of multi-hop communication the temperature aware routing protocol M-ATTEMPT minimize the energy consumption and temperature leap. This protocol has the increased packet delivery ratio the energy consumption and temperature leap. This protocol has the increased packet delivery ratio.

A cross layered protocol for WBANs is WASP [20] which enhance the packet delivery ratio. Other benefits of WASP includes, it minimizes the energy absorption of nodes and in addition to this also reduces the end-to-end delay. Like other protocols it also has some drawbacks, which has its poor link established quality, and it does not upkeep shared communication. An improve variation of WASP is CICADA [22], also a cross layered protocol. CICADA has sufficient sleep time as compare to WASP; consequently it declines energy consumption for sensor nodes. DARE [23] consumes less energy due to sink nodes attach on chest of each patient. This protocol presents five different scenarios for placements of node on patient's body, which results in longer network life time and greater stability period. But these scenarios make DARE owning high propagation delay due to its distance with sink.

The opportunistic protocol [24] is a postural movement based routing protocol, having the whole energy consumption amongst single hop and multi-hop communication. It has the equal level of energy consumption for relay node and for sensor node i.e $50 \%$ for relay and $50 \%$ for sensor nodes. In opportunistic protocol a separable energy level is not measured; however as the number of nodes rise in network the circulation load on the relay node will automatically increases. HIT [25] absorbs much power for thick or large network. However, to establish a direct link for lesser number of sensor nodes in a network, HIT performs well for minimum energy consumption. Additionally, it is beneficial for data gathering due to its less network delay. HIT does not bother packet delivery latency and shows reduce packet delivery delay and energy absorption. MIEEPB [26] is an extended version of improved energy efficient PEGASIS-based protocol (IEEPB), with the idea of mobile sink. MIEEPB has provided an optimal approach and enhance the network lifetime as compared to previous version. In [27] authors are evaluating the performance of IEEE 802.15.4 for multi-hop transmission in low power sensor network by varying data rates and frequencies. Authors have done this by under variable frequencies and data rates.

Table 1. Comparison of routing Protocols

\begin{tabular}{|l|l|l|l|l|l|l|l|}
\hline \multirow{2}{*}{$\begin{array}{l}\text { Charact } \\
\text { eristics }\end{array}$} & \multicolumn{5}{|c|}{ Protocols } \\
\cline { 2 - 8 } & $\begin{array}{l}\text { M- } \\
\text { MPTE }\end{array}$ & WASP & $\begin{array}{l}\text { CICA } \\
\text { DA }\end{array}$ & DARE & $\begin{array}{l}\text { Oppor } \\
\text { tunisti } \\
\text { c }\end{array}$ & HIT & $\begin{array}{l}\text { MIE } \\
\text { EPB }\end{array}$ \\
\hline $\begin{array}{l}\text { Network } \\
\text { Lifetime }\end{array}$ & $\begin{array}{l}\text { Norma } \\
\text { PDR }\end{array}$ & High & High & High & High & $\begin{array}{l}\text { Nor } \\
\text { mal }\end{array}$ & $\begin{array}{l}\text { Ver } \\
\text { High } \\
\text { Hig }\end{array}$ \\
\hline $\begin{array}{l}\text { Energy } \\
\text { cons. }\end{array}$ & Low & Low & $\begin{array}{l}\text { Very } \\
\text { Low }\end{array}$ & Low & $\begin{array}{l}\text { Very } \\
\text { Low }\end{array}$ & Low & $\begin{array}{l}\text { Nor } \\
\text { mal }\end{array}$ \\
\hline Delay & Low & Low & $\begin{array}{l}\text { Very } \\
\text { Low }\end{array}$ & High & Low & Low & Low \\
\hline Mobility
\end{tabular}

\section{CONCLUSION}

WBSN technology offers the prototype towards proactive management by concentrating on anticipation and pre detection of different types of diseases. This can bring revolution in the coming generation health care concerns and decreases the cost of health related issues. Due to distinctive in-body and on - body constraints designing a routing protocol for WBASNs is a challenging task. The suggested routing protocols either do not take into consideration postural body movements with mobility or are not as energy efficient. Also, many of the above mentioned routing protocols did not painstaking reliability and QoS.

In future, routing protocols for WBASNs must provide longer network life through energy efficient schemes. They should provide energy efficient and reliable communication between sensor nodes in real-time and non-real-time application. A reliable communication among heterogeneous bio-medical sensor nodes in real time application is also needed. They must take into consideration the issues of Latency, temperature effects, reliability and power consumption for developing better routing protocol models of WBANs. Different routing challenges belongs to WBASNs are measured in different categories of routing protocols , but still lots of work needs to be done in this regard. Moreover, much accurate and proficient network architecture needs to be developed for best routing structure in WBSNs. As Future work, routing protocols for BANs must be capable of gaining the required QoS as well as preserving well-balanced low power energy consumption. These goals can be gained by mutually construction the Mac layer and routing protocols. 


\section{REFERENCES}

[1] Yick, J.; Mukherjee, B.; Ghosal, D. Wireless sensor network survey. Comput. Netw. 2008, 52, 2292-2330.

[2] Mainwaring, Alan, et al. "Wireless sensor networks for habitat monitoring." Proceedings of the 1st ACM international workshop on Wireless sensor networks and applications. ACM, 2002.

[3] N. Javaid, Z. Abbas, M. S. Farid, Z. A. Khan and N. Alrajeh, "M-ATTEMPT: A New Energy-Efficient Routing Protocol for Wireless Body Area Sensor Networks", in proc. of 4th International Conference on Ambient Systems, Networks and Technologies (ANT 2013), Pages 224-231, 2013.

[4] United Nations. World Population Prospects: The 2012 Revision. Available online: http://esa.un.org/wpp/ (accessed on 13 June 2014).

[5] Oey, C.H.W.; Moh, S. A Survey on temperature-aware routing protocols in wireless body sensor networks. Sensors 2013, 13, 9860-9877.

[6] Chen, C.; Knoll, A.; Wichmann, H-E.; Horsch, A. A review of three-layer wireless body sensor network systems in healthcare for continuous monitoring. J. Mod. Internet Things 2013, 2, 24-34.

[7] Milenković, A.; Otto, C.; Jovanov, E. Wireless sensor networks for personal health monitoring: Issues and an implementation. Comput. Commun. 2006, 29, 25212533.

[8] N. Javaid, U. Qasim, Z. A. Khan, M. A. Khan, K. Latif and A. Javaid, "On Energy Efficiency and Delay Minimization in Reactive Protocols in Wireless Multihop Network", 2nd IEEE Saudi International Electronics, Communications and Photonics Conference (SIECPC 13), 2013, Riyadh, Saudi Arabia.

[9] B. Manzoor, N. Javaid, O. Rehman, M. Akbar, Q. Nadeem, A. Iqbal, M. Ishfaq, "Q-LEACH: A New Routing Protocol for WSNs", in proc. of 4th International Conference on Ambient Systems, Networks and Technologies (ANT 2013), Procedia Computer Science, Volume 19, 2013, Pages 926-931, ISSN 1877 0509

[10] N. Javaid, R. D. Khan, M. Ilahi, L. Ali, Z. A. Khan, U. Qasim, "Wireless Proactive Routing Protocols under Mobility and Scalability Constraints", J. Basic. Appl. Sci. Res., 3(1)1187-12001, 2013

[11] B. Latre, B. Braem, I. Moerman, C. Blondia, and P. Demeester, "A survey on wireless body area networks," Wirel. Netw. vol. 17, pp. 1-18, Jan. 2011.

[12] C. Tachtatzis, F. Di Franco, D. Tracey, N. Timmons, and J. Morrison, "An energy analysis of IEEE 802.15.6 scheduled access modes," IEEE GLOBECOM, pp. 1270-1275, 2010.

[13] R. Shah and M. Yarvis, "Characteristics of on-body 802.15.4 networks," 2nd IEEE Workshop on Wireless Mesh Networks, pp. 138 -139, Sep. 2006.

[14] D. Domenicali and M.-G. Di Benedetto, "Performance analysis for a body area network composed of IEEE 802.15.4a devices," $4^{\text {th }}$ Workshop on Positioning, Navigation and Communication (WPNC'07), pp. $273-$ 276, Mar. 2007.
[15] Movassaghi, S.; Abolhasan, M.; Lipman, J. "A review of routing protocols in wireless body area networks" J. Netw. 2013, 8, 559-575

[16] J. I. Bangash, A. H. Abdullah, M. H. Anisi, A. W. Khan, "A Survey of Routing Protocols in Wireless Body Sensor Networks", Sensor Vol. 14, No.1, 2014.

[17] Q. Tang, N. Tummala, Thermal-aware routing algorithm for implanted sensor networks, in: Proceedings of the International Conference on Distributed Computing in Sensor Systems, 2005, pp. 206217.

[18] M. Quwaider, S. Biswas, On-body packet routing algorithms for body sensor networks, in: Networks and Communications, 2009. NETCOM 09. First International Conference on, 2009, pp. 171177.

[19] D. Y. Kim, W. Y. Kim, J. S. Cho, B. Lee, Ear: An environment-adaptive routing algorithm for WBANs, in Proc. of International Symposium on Medical Information and Communication Technology (ISMICT) 2010.

[20] B. Braem, B. Latre, I. Moerman, C. Blondia, P. Demeester, "The wireless autonomous spanning tree protocol for multihop wireless body area networks", in Proc. of First International Workshop on Personalized Networks, 2006.

[21] R. Annur, N. Wattanamongkhol, N. Nakpeerayuth, L. Wuttisittikulkij, J. Takada, "Applying the tree algorithm with prioritization for body area networks", in 10th International Symposium on Autonomous Decentralized Systems, 2011.

[22] Latre, Benoit, et al. "A low-delay protocol for multihop wireless body area networks." In proc. of $4^{\text {th }}$ IEEE Int'l Conf. on Mobile and Ubiquitous Systems: Networking and Services, 2007

[23] A. Tauqir, N. Javaid, S. Akram, A. Rao, S. N Mohammad " Distance Aware Relaying Energyefficient: DARE to Monitor Patients in Multi-hop Body Area Sensor Networks" IEEE Eighth International Conference on Broadband and Wireless Computing, Communication and Applications (BWCCA), 26 July 2013.

[24] Maskooki, A.; Soh, C.B.; Gunawan, E.; Low, K.S Opportunistic Routing for Body Area Networks. In proc. of IEEE Consumer Communications and Networking Conference, USA, 2011, pp. 237-241.

[25] Culpepper, B.J.; Dung, L.; Moh, M. Design and analysis of Hybrid Indirect Transmissions (HIT) for data gathering in wireless micro sensor networks. ACM SIGMOBILE Mobile Comput. Commun. Rev. 2004, 8, $61-83$

[26] M. R. Jafri. Javaid, A. Javaid, Z.A Khan, "Maximizing the lifetime of multi-chain pegasis using sink mobility" World Applied Sciences Journal, Vol. 2 ,arXiv preprint arXiv:1303.4347.201

[27] M. Mateen Yaqoob, et al. "Examination of IEEE 802.15.4 ZigBee Multi-Hop Transmission in Wireless Body Area Networks", ITEE Journal, Vol. 2 No. 1, pp. 33-37, 2013. 